\title{
张掖湿地芨芨草叶大小和叶脉密度的权衡关系
}

\author{
韩 玲 赵成章 ${ }^{*}$ 徐 婷 冯 威 段贝贝 郑慧玲
}

西北师范大学地理与环境科学学院, 甘肃省湿地资源保护与产业发展工程研究中心, 兰州 730070

摘 要 叶大小-叶脉密度的权衡关系是植物叶经济谱理论的基础, 对理解资源竞争条件下植物叶片的物理构建与生理代谢 的关系具有重要的意义。该文采用标准化主轴估计(standardized major axis estimation, SMA)的方法, 按菠茂草(Achnatherum splendens)株从密度设置I $\left(>12\right.$ 从 $\left.\cdot \mathrm{m}^{-2}\right) 、$ II $\left(8-12\right.$ 从 $\left.\cdot \mathrm{m}^{-2}\right) 、$ III $\left(4-8\right.$ 从 $\left.\cdot \mathrm{m}^{-2}\right)$ 和IV $\left(<4\right.$ 从 $\left.\cdot \mathrm{m}^{-2}\right) 4$ 个密度梯度, 以叶面积和叶干质量分 别表示叶大小, 对张掖洪泛平原湿地不同密度条件下芨芨草种群的叶大小和叶脉密度的关系进行研究。结果表明: 随着芨芨 草株从密度的降低, 湿地群落的土壤含水量逐渐减小、土壤电导率逐渐增加, 芨芝草的净光合速率 $\left(P_{\mathrm{n}}\right)$ 、蒸滕速率 $\left(T_{\mathrm{r}}\right)$ 和分枝 数呈先增大后减小的趋势, 叶面积、叶干质量、比叶面积和株高呈逐渐减小趋势、光合有效辐射 $(P A R)$ 和叶脉密度呈逐渐增 加趋势; 菆菆草叶大小和叶脉密度在高密度(I)和低密度(IV)样地均呈极显著负相关关系 $(p<0.01)$, 中密度(II、III)样地二者呈 显著负相关关系 $(p<0.05)$; 叶大小和叶脉密度回归方程的SMA斜率在不同密度样地均显著小于-1 $(p<0.05)$, 即茂茂草叶大 小和叶脉密度呈“此消彼长”的权衡关系。在高密度湿地群落芨芝草倾向于大叶片低叶脉密度的叶片构建模式, 在低密度湿地 群落选择小叶片高叶脉密度的异速生长模式, 体现了密度制约下湿地植物的生物量分配格局和资源利用对策。

关键词 叶面积; 叶干质量; 叶脉密度; 权衡; 茂菆草; 张掖湿地

引用格式: 韩玲, 赵成章, 徐婷, 冯威, 段贝贝, 郑慧玲 (2016). 张掖湿地芨芨草叶大小和叶脉密度的权衡关系. 植物生态学报, 40, 788-797. doi: 10.17521/cjpe.2016.0003

\section{Trade-off between leaf size and vein density of Achnatherum splendens in Zhangye wetland}

\author{
HAN Ling, ZHAO Cheng-Zhang*, XU Ting, FENG Wei, DUAN Bei-Bei, and ZHENG Hui-Ling \\ College of Geography and Environmental Science, Northwest Normal University, Research Center of Wetland Resources Protection and Industrial Develop- \\ ment Engineering of Gansu Province, Lanzhou 730070, China
}

\section{Abstract}

Aims Trade-offs between leaf size and vein density are the basis of the theory of leaf economics spectrum, and are to understand the relationship between the physical build and physiological metabolism of plant leaves under different degrees of competition for resources. Our objective was to study the changes in the relationship between leaf size and vein density (leaf dry biomass and leaf area) in Achnatherum splendens populations with four plant bundle densities located in the flood plain wetland of Zhangye.

Methods The study site was located at floodplain wetlands of Zhangye, Gansu Province, China. Survey and sampling were carried out in the communities that A. splendens dominated. According to the plant bundle density, the A. splendens communities were divided into four density gradients with "bundle" for the sampling units, high density (I, > 12 bundle $\cdot \mathrm{m}^{-2}$ ), medium density (II, 8-12 bundle $\cdot \mathrm{m}^{-2}$ ), medium density (III, 4-8 bundle $\cdot \mathrm{m}^{-2}$ ) and Low density (IV, $<4$ bundle $\left.\cdot \mathrm{m}^{-2}\right)$. According to the density of each combination, we chose seven $(5 \mathrm{~m} \times 5 \mathrm{~m}) A$. splendens samples, resulting in a total of 28 samples $(4 \times 7)$. The soil physical and chemical properties of four density gradients were investigated and six samples of $A$. splendens were used to measure the leaf area, leaf dry biomass and vein density in laboratory, and biomass of different organs was measured after being dried at $85{ }^{\circ} \mathrm{C}$ in an oven. 28 plots were categorized into three groups: high, medium and low density, and the standardized major axis (SMA) estimation method was used to examine the allometric relationships between leaf area, leaf dry biomass and vein density.

Important findings The results showed that with the population density changed from high, medium, to low, the soil moisture decreased, and soil electric conductivityincreased. The leaf area, leaf biomass and height of $A$. splendens decreased, and the vein density, specific leaf area and photosynthetically active radiation (PAR)

收稿日期Received: 2016-01-03 接受日期Accepted: 2016-05-12

* 通信作者Author for correspondence (E-mail: zhaocz601@163.com) 
increased gradually. In addition, leaf net photosynthetic rate $\left(P_{\mathrm{n}}\right)$, transpiration rate $\left(T_{\mathrm{r}}\right)$ and twig number firstly increased then decreased. There was a highly significantly negative correlation $(p<0.01)$ between the leaf size and vein density on the high- and low-level densities (I, IV), whereas less significant $(p<0.05)$ on the level of medium density (II, III). The SMA slope of regression equation in the scaling relationships between leaf size and vein density was significantly smaller than $-1(p<0.05)$.

Key words leaf area; leaf dry biomass; vein density; trade-off; Achnatherum splendens; Zhangye wetland

Citation: Han L, Zhao CZ, Xu T, Feng W, Duan BB, Zheng HL (2016). Trade-off between leaf size and vein density of Achnatherum splendens in Zhangye wetland. Chinese Journal of Plant Ecology, 40, 788-797. doi: 10.17521/cjpe.2016.0003

植物是一个功能和结构平衡的生命体, 在漫长 的进化过程中通过逐步调整结构, 使其功能和结构 达到权衡状态, 这对植物提高光合收益和优化生存 策略具有重要的意义(Brodribb \& Jordan, 2011; Reich \& Cornelissen, 2014)。叶片形态及叶脉网络功 能性状能够反映植物适应特定生境的基本行为和光 合生理功能, 体现了植物提高叶片光截取、碳获取 以及水分输导效率的生态策略(金鹰和王传宽, 2015)。叶脉为广泛分布于整个叶片的网络系统结构 (Brodribb et al., 2010), 叶脉密度反映了叶脉与叶肉 的接触程度和叶片的碳投资模式, 其大小影响光合 蒸腾过程中水分、养分及光合产物运输的效率与安 全性(Sack \& Frole, 2006; Sack \& Scoffoni, 2013); 叶 大小的变化与某一给定的叶生物量分配和总叶面积 密切相关(Niklas et al., 2007), 决定了植物对光的拦 截效率和碳获取能力(王常)顺和汪诗平, 2015), 直接 影响着植物光合生产力和生存适合度(Castro-Díez et al., 2000); 比叶面积是一定干物质投资所展开的 捕光表面积数量, 对植物的相对生长速率具有重要 影响(Wright et al., 2004)。叶片结构性状和光合生理 活性间相互联系、相互依存的内在关系, 反映了植 物的结构特性和生物学过程对资源利用水平的决定 作用(金鹰和王传宽, 2015)。随着叶片的展开, 植物 的净光合速率 $\left(P_{\mathrm{n}}\right)$ 和叶片蒸腾速率 $\left(T_{\mathrm{r}}\right)$ 会随之增加 (Sack et al., 2012), 较高的叶脉密度能够满足蒸腾 带来的大量水分和能量消耗, 有助于植物调节叶片 温度和维持体内水分平衡(Nardini et al., 2010), 有 限的光合产物不可能同时兼顾叶肉、叶脉等组织间 的资源投入, 导致在某种情况下叶脉密度又会限制 叶面积和光合组织密度最大化, 反映了叶片蒸腾成 本与光合收益之间的相互匹配与经济权衡关系 (Sack \& Scoffoni, 2013), 对植株构建最佳的生理和 功能状态发挥着重要的调控作用。因此, 叶大小与 叶脉密度之间相互影响、相互制约的生态学机制是
理解植物生理活动中叶片功能多样化和光合碳固定 的重要依据(杨冬梅等, 2012; 李乐等, 2013)。密度是 自然界植物种群普遍存在的一种重要选择压力 (Japhet et al., 2009), 随着种群密度的增加, 植物生 存所需空间、光照和土壤水分等资源可能受到邻株 的物理或几何上的拥挤效应，从而引起不同程度的 隐蔽度和竞争强度的干扰(张明娟等, 2012), 改变了 种群内每个植株可获得性资源的数量, 植物的光合 作用将被抑制(史元春等, 2015), 促使植物权衡叶大 小和叶脉密度的生物量分配、调整叶片构型构建, 实现叶片生理特性和环境空间异质性的精细协调, 体现了最大化地吸取异质性分布资源的受食行为和 可塑性反应, 也是植物最经济的生境适应性和风险 规避对策(Silvertown \& Charlesworth, 2001)。因此, 研究不同密度间植物叶大小和叶脉密度的关系及成 因, 对于理解干旱区湿地群落密度制约下植物叶经 济谱的形成机制具有重要意义。

芨菆草(Achnatherum splendens)是我国西北地 区常见的多年生密丛性草本植物, 草秆坚硬、直立 丛生、根系发达、须根较多, 叶片拥有较强的机械 组织和输导组织, 具有较强的耐旱、耐盐碱的表型 可塑性, 通常发育于轻度盐化的河湖边缘以及低洼 地等隐域性生境, 是一种优良的饲用植物、纤维植 物和水土保持植物(张翼飞等, 2012)。近年来, 学术 界对植物叶大小的形成机制和环境适应性 (Mcdonald et al., 2003; 刘明虎等, 2013; 党晶晶等, 2014)、叶脉网络功能性状与叶片光合(Brodribb et al., 2010; Sack et al., 2012)、水分利用能力(Zhang et al., 2012; Sack \& Scoffoni, 2013)、碳构建(Reich \& Cornelissen, 2014)的关系以及叶脉密度与叶大小的 比例关系(sack et al., 2012)等已有大量研究, 而叶片 各功能性状间关联性的形成可能依赖于不同研究尺 度和环境因子的差异。目前对芨芨草的研究集中在 不同密度芨菆草空间格局对环境胁迫的响应(张明 
娟等, 2012)、生物学特征和抗盐性(张雅琼等, 2010) 及水分利用策略(Wang et al., 2004)等方面, 但芨菆 草叶片形态和叶脉性状的生长规律研究不深入, 尤 其湿地群落不同密度生境叶大小和叶脉密度的生长 关系对植物生理功能和异质性资源配置的响应机理 尚不清晰。鉴于此, 本文用叶面积和叶干质量分别 表示叶大小, 研究张掖湿地群落不同密度间芨芨草 叶大小-叶脉密度的关系及差异, 旨在理解密度制 约下植物叶片的物理构建方式, 有助于揭示湿地异 质生境中植物功能结构耦合与权衡的生理生态学机 制。

\section{1 材料和方法}

\section{1 研究区域和样地概况}

研究区位于甘肃省张掖市甘州区西城驿黑河干 流河床边缘缓坡地带的洪泛平原湿地, 地理位置为 $38.56^{\circ} \mathrm{N}, 100.26^{\circ} \mathrm{E}$, 海拔 $1482.7 \mathrm{~m}$, 属温带大陆性 气候, 雨热同期, 年平均气温 $7.8{ }^{\circ} \mathrm{C}, \geqslant 0{ }^{\circ} \mathrm{C}$ 积温为 $2734{ }^{\circ} \mathrm{C}$, 年降水量132.6 mm, 主要集中在6-9月, 年蒸发量 $1986.5 \mathrm{~mm}$, 年日照时间 $3077 \mathrm{~h}$, 土壤以 砾石土、灰棕荒漠土和草甸土为主, 湿地内土壤类 型的多样化决定了旱生、盐生、湿生等植物群落的 形成, 植被分布具有明显的水平分异特征, 以湿生 植物、盐生植物与陆生乔灌林木为主。主要植物有 芨茂草、芦苇(Phragmites australis)、冰草(Agropyron cristatum)、节节草(Equisetum ramosissimum)、宽苞 水柏枝 (Myricaria bracteata)、多枝柽柳(Tamarix ramosissima)和沙柊(Elaeagnus angustifolia)等。该研 究区属于张掖黑河国家湿地保护区核心区, 不存在 家畜放牧和人类活动等干扰因素, 在丰水期季节性 和间歇性洪水能够满足植物生长的水分需求, 并局 部改善湿地的水盐状况; 在枯水期除一些洼地存有 水塘外, 大部分区域没有地表水, 植被主要依靠地 下潜水生存。在降水稀少、蒸发强烈的大环境影响 下, 湿地土壤的盐渍化程度高, 土壤水分空间分异 明显, 湿地植被群落的结构组成单一、物种多样性 低、寡种优势现象较为普遍, 菆菆草凭借较强的生 态可塑性, 形成了不同于河岸林、河流湿地和荒漠 的洪泛湿地单优势种群植被景观(占玉芳等, 2012)。

\section{2 实验材料}

芨芨草为多年生草本植物, 与典型盐生植物相 比, 芨芨草并非喜盐植物, 仅是生态幅较宽的耐盐
旱生植物, 在海拔较低、地下水位较高的碱性湿地 平原以至高达5 $000 \mathrm{~m}$ 的青藏高原, 从干草原带一 直到荒漠区均有分布(张雅琼等, 2010)。叶丛茂盛, 叶鞘无毛, 较疏松, 具膜质边缘; 叶舌三角形或尖 披针形, 长5-10 mm; 叶片扁平或边缘内卷, 质坚 㓞, 长30-60 cm, 宽7-12 mm, 叶片上表皮可见6-7 条沿大小脉呈深波状脉纹突起, 微粗䊁有表皮毛, 叶脉大小维管束相间排列, 无主脉, 叶片的中脉与 侧脉平行排列, 维管束鞘两层, 下表皮光滑无毛(吴 征镒和郭本兆, 1989)。

\section{3 实验设置}

\subsection{1 植物采样}

2015年8月初, 在实地考察的基础上, 选择一块 芨芨草为优势种的湿地群落, 以“从”为取样单位进 行实验, 按芨芨草株从(即由种子产生, 由无性繁殖 而生成的无性系)的密度状况(张明娟等, 2012), 将 芨芨草株从的密度水平分为 4 个梯度, 分别为: 高 密度 $\left(\mathrm{I},>12\right.$ 从 $\left.\cdot \mathrm{m}^{-2}\right)$ 、中密度(II, $8-12$ 从 $\left.\cdot \mathrm{m}^{-2}\right)$ 和(III, $4-8$ 从 $\left.\cdot \mathrm{m}^{-2}\right)$ 、低密度 $\left(\mathrm{IV},<4\right.$ 从 $\left.\cdot \mathrm{m}^{-2}\right)$ 。按已划分的苃 芨草种群的密度梯度各选取 7 个 $5 \mathrm{~m} \times 5 \mathrm{~m}$ 样方, 总 计 28 个样方。首先在每一个样方各选择大中小 2 从夰 芨草, 共6丛, 用卷尺测量从基部到最高分枝的高 度, 定位株高, 并记录芨芨草株从分枝数, 然后选 取每个株从最外层 4 个方位充分伸展且健康完整的 2-3个叶片, 做好标记后进行以下步骤:

(1)植物光合参数测定。于 8月15-18日(代表植物 生长旺盛期), 选择晴朗天气的9:00-12:00进行气体 交换参数的测定。光合测定使用GFS-3000便携式光 合测量系统(Heinz Walz GmbH, Bavaria, Germany), 测量过程中使用人工红蓝光源, 光合有效辐射 $(P A R)$ 为 $1200 \mu \mathrm{mol} \cdot \mathrm{m}^{-2} \cdot \mathrm{s}^{-1}, \mathrm{CO}_{2}$ 浓度约为 340 $\mu \mathrm{mol} \cdot \mathrm{mol}^{-1}$, 相对湿度 $(R H)$ 保持在 $40 \%-50 \%$, 流速 设定为 $750 \mu \mathrm{mol} \cdot \mathrm{s}^{-1}$, 叶室面积为 $8 \mathrm{~cm}^{2}$, 对做好标记 的每个叶片记录 5 组数据用于统计分析, 分别测定 叶片的 $P_{\mathrm{n}} 、 T_{\mathrm{r}}$ 等参数(任青吉等, 2015), 对于不能充 满叶室的叶片, 则保存于湿润的封口袋中, 带回实 验室, 用便携式激光叶面积仪(CI-202, Walz, Camas, USA)测定叶面积, 进而计算 $P_{\mathrm{n}}$ 和 $T_{\mathrm{r}}$ 。

(2) 选择晴朗无云的天气用手持光量子计 (3415F, 3415F, Walz, Plainfield, USA)于9:00-11:00 对 4 个密度梯度内所有芨芨草进行PAR测定, 测定位 置分别在植株上方(距冠层 $15 \mathrm{~cm}$ )、中层(株高 $1 / 2$ 处) 
和地表(距地表 $15 \mathrm{~cm}$ 处)。

(3)叶脉密度和叶片形态特征的测定。基于每个 芨芨草被标记的2-3个叶片, 采集后保存于湿润的 封口袋中, 带回实验室用福尔马林-乙酸溶液 $(37 \%$ 甲醛溶液、50\%乙醇和 $13 \%$ 冰醋酸溶液)固定。每片 叶片先测量叶脉密度数据, 用含 $5 \% \mathrm{NaOH}$ 的乙醇进 行化学清理, 再用番红-固绿染色(Berlyn \& Miksche, 1976), 用水包埋呈透明薄膜状在体视显微镜 (SMZ168-BL, Motic, Hong Kong, China)下放大10倍 后拍照, 每个叶片拍摄 10 个视野; 再用Motic Images Plus 2.0软件统计分析叶脉的总长度, 叶脉密度 用单位叶面积(单位: $\mathrm{mm}^{2}$ )的叶脉总长度(单位: $\mathrm{mm}$ ) 表示(Sack et al., 2012); 最后将叶片放在 $80{ }^{\circ} \mathrm{C}$ 的烘 箱中烘干48 h 至恒质量, 用电子天平(精度为 0.0001 g)称取质量, 记录叶干质量。

\subsection{2 土壤取样与理化性质测量}

(1)土壤含水量采样。土壤取样于2015年8月下 旬完成, 取样时间内基本无明显降水, 土壤水盐状 况相对稳定。在每个小样方随机选取 3 个样点挖掘 1 $\mathrm{m} \times 1 \mathrm{~m} \times 0.5 \mathrm{~m}$ 土壤剖面, 用环刀分5层间隔 $10 \mathrm{~cm}$ 采取土样, 现场编号、称鲜质量后带回实验室在105 ${ }^{\circ} \mathrm{C}$ 的烘箱内烘 $12 \mathrm{~h}$, 取出称质量, 计算出各样地 0-50 cm土层土壤质量含水量。

(2) 土壤盐分采样。用电导法测定土壤含盐量, 在室温下称取过 $2 \mathrm{~mm}$ 篎的风干土样 $10 \mathrm{~g}$, 加 $50 \mathrm{~mL}$ 去 $\mathrm{CO}_{2}$ 蒸馏水 (水土比为5:1), 取浸出液, 置振荡机 上振荡 $5 \mathrm{~min}$ 。将布氏漏斗与抽气系统相连后把悬浊 的土浆缓缓倒入漏斗, 直至抽滤完毕, 滤液倒入三 角瓶备用(张雅琼等, 2010)。用上海雷磁仪器厂生产 的DDS-11C便携式电导仪测浸出液的电导率, 3次重 复, 取平均值。

\section{4 数据分析}

对芨菆草种群样地划分出的 4 个密度梯度进行 各性状统计分析。根据各密度梯度已测定的叶面积 和叶干质量的平均值, 计算出菆菆草的比叶面积, 计算公式为: 比叶面积(单位: $\mathrm{cm}^{2} \cdot \mathrm{g}^{-1}$ ) $=$ 叶面积/叶 干质量。芨芨草叶大小分别指单叶面积和单叶干质 量, 对芨芨草叶大小和叶脉密度的实验数据先进行 以 10 为底的对数转换, 使之符合正态分布后再进行 分析。对任意两个功能关系的研究, 我们采用 $y=$ $a x^{b}$, 线性转换成 $\log (y)=b \log (x)+\log (a)$, 式中 $x$ 和 $y$ 表示两个特征参数, $b$ 为斜率, 即异速生长参数或相
对生长的指数, 当 $|b|=1$ 时, 表示两者是等速变化关 系; 当 $|b|$ 显著偏离 1 时, 两者间为异速变化关系 (Harvey \& Pagel, 1991)。密度间异速生长方程的参 数估计, 主要采用标准化主轴估计 (SMA) 方法 (Warton et al., 2006), 由软件(S)MATR Version 2.0 (Falster et al., 2012)计算完成, 每一个回归斜率的置 信区间根据Pitman (1939)方法计算，并采用Warton 和Weber (2002)的方法对每个密度的斜率进行异质 性测试。不同密度间植物功能性状平均值的差异比 较采用单因素方差分析 (one-way ANOVA, $\alpha=$ $0.05)$, 实验所用数据采用SPSS 16.0 软件进行处理 分析, 用SigmaPlot 10.0和Excel软件绘图。

\section{2 结果分析}

\section{1 不同密度条件下湿地䓅芨草光合生理和生物 学特征变化}

不同密度条件下菆芨草的光合生理和生物学特 征的变化如表1所示。随着芨芨草株丛密度的逐渐降 低, 芨芨草的株高呈逐渐减小趋势, 从密度(I)到密 度(IV)减少了 $18.84 \%$, 中密度(II、III)无显著差异 $(p$ $>0.05)$; 芨芨草分枝数、 $P_{\mathrm{n}}$ 和 $T_{\mathrm{r}}$ 呈先增大后减小的趋 势, 从高密度 (I) 到低密度 (IV), 分枝数增加了 $45.89 \%, P_{\mathrm{n}}$ 和 $T_{\mathrm{r}}$ 从高密度(I)到低密度(IV)分别减少 $12.65 \%$ 和 $7.44 \%$, 中密度(II、III)均出现较大值且无 显著差异 $(p>0.05)$; 芨芨草的 $P A R$ 呈逐渐增大趋势, 由高密度(I)的 $636.30 \mu \mathrm{mol} \cdot \mathrm{m}^{-2} \cdot \mathrm{s}^{-1}$ 增加到低密度(IV) 的1 $105.10 \mu \mathrm{mol} \cdot \mathrm{m}^{-2} \cdot \mathrm{s}^{-1}$, 增加了 0.74 倍, 不同密度 条件下菆芨草 $P A R$ 存在显著差异 $(p<0.05)$ 。

\section{2 不同密度条件下湿地芨芨草叶性状和土壤理 化性质的变化}

不同密度条件下菆芨草种群叶性状和土壤理化 性质的变化如图1所示。由单因素方差分析得出, 夰 夰草叶面积、叶干质量和土壤电导率在不同密度存 在显著差异 $(p<0.05)$, 叶脉密度、比叶面积和土壤 含水量在中密度(II、III)无显著差异 $(p>0.05)$ 。随着 芨芨草株从密度的逐渐降低, 芨芨草叶面积和叶干 质量呈逐渐减小趋势, 叶脉密度呈逐渐增大趋势, 从高密度(I)到低密度(IV), 叶面积和叶干质量分别 减小了 $47.18 \%$ 和 $40 \%$ 、叶脉密度增加了 $13.33 \%$; 苃 芨草比叶面积随株从密度的降低呈逐渐减小趋势, 从最大值到最小值减小了 $12.5 \%$; 土壤电导率随密 度的降低呈逐渐增加趋势, 从高密度 $(\mathrm{I})$ 的 3.14 
表1 不同密度条件下芨芨草种群光合生理和生物学特征(平均值土标准误差)

Table 1 Photosynthetic physiological and biological characteristics of Achnatherum splendens under different densities (mean \pm SE)

\begin{tabular}{|c|c|c|c|c|c|}
\hline $\begin{array}{l}\text { 密度 } \\
\text { Density }\end{array}$ & $\begin{array}{c}\text { 株高 } \\
\text { Plant high (cm) }\end{array}$ & $\begin{array}{c}\text { 分枝数 } \\
\text { Twig number }\end{array}$ & $\begin{array}{c}P_{\mathrm{n}} \\
\left(\mu \mathrm{mol} \mathrm{CO}{ }_{2} \cdot \mathrm{m}^{-2} \cdot \mathrm{s}^{-1}\right)\end{array}$ & 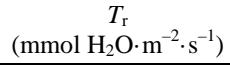 & $\begin{array}{c}P A R \\
\left(\mu \mathrm{mol} \cdot \mathrm{m}^{-2} \cdot \mathrm{s}^{-1}\right)\end{array}$ \\
\hline$>12$ bundle $\cdot \mathrm{m}^{-2}(\mathrm{I})$ & $161.00 \pm 10.24^{\mathrm{a}}$ & $113.33 \pm 5.29^{\mathrm{d}}$ & $13.20 \pm 0.12^{\mathrm{c}}$ & $6.45 \pm 0.02^{\mathrm{c}}$ & $636.30 \pm 14.18^{\mathrm{d}}$ \\
\hline $8-12$ bundle $\cdot \mathrm{m}^{-2}$ (II) & $149.67 \pm 8.62^{\mathrm{b}}$ & $203.67 \pm 12.34^{\mathrm{b}}$ & $13.83 \pm 0.17^{\mathrm{a}}$ & $6.58 \pm 0.09^{\mathrm{a}}$ & $839.20 \pm 27.95^{c}$ \\
\hline 4-8 bundle $\cdot \mathrm{m}^{-2}$ (III) & $140.34 \pm 7.50^{\mathrm{b}}$ & $273.33 \pm 15.83^{\mathrm{a}}$ & $14.12 \pm 0.18^{\mathrm{a}}$ & $6.67 \pm 0.10^{\mathrm{a}}$ & $918.80 \pm 36.94^{\mathrm{b}}$ \\
\hline$<4$ bundle $\cdot \mathrm{m}^{-2}$ (IV) & $130.67 \pm 5.56^{\mathrm{C}}$ & $165.33 \pm 11.90^{\mathrm{c}}$ & $13.77 \pm 0.13^{\mathrm{b}}$ & $6.53 \pm 0.03^{b}$ & $1105.10 \pm 40.62^{\mathrm{a}}$ \\
\hline
\end{tabular}

$P A R$, 光合有效辐射; $P_{\mathrm{n}}$, 净光合速率; $T_{\mathrm{r}}$, 蒸腾速率。同列不同小写字母表示不同密度间差异显著 $(p<0.05)$ 。

$P A R$, photosynthetically active radiation; $P_{\mathrm{n}}$, net photosynthetic rate; $T_{\mathrm{r}}$, transpiration rate. Different lowercase letters in the same column indicate significant differences of different density treatments $(p<0.05)$.
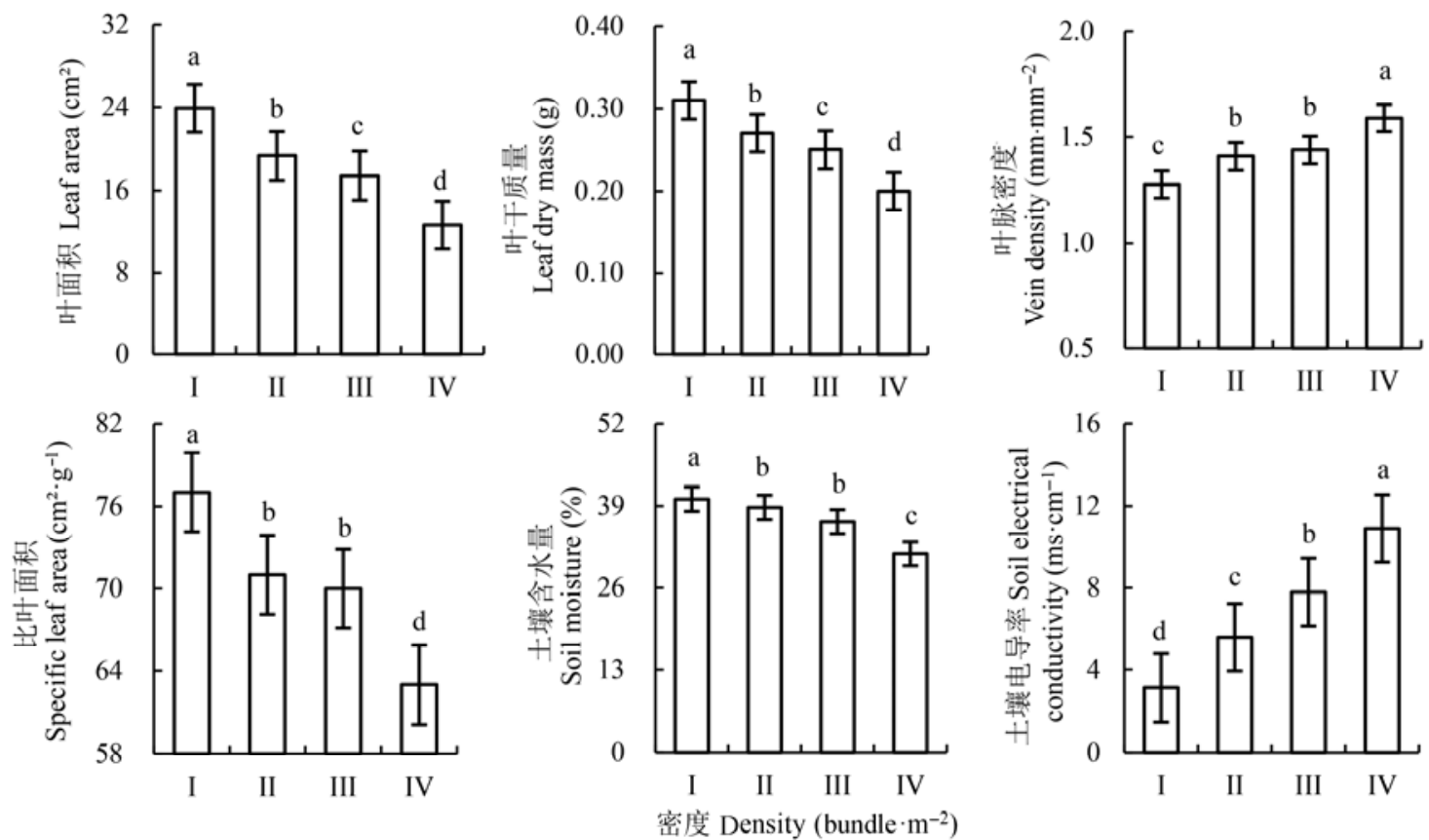

图1 不同密度条件下芨芨草叶性状和土壤理化性质的变化(平均值土标准误差)。不同小写字母表示相同构件在不同密度处理 下差异显著 $(p<0.05)$ I，高密度 $\left(>12\right.$ bundle $\left.\cdot \mathrm{m}^{-2}\right)$; II, 中密度 $\left(8-12\right.$ bundle $\left.\cdot \mathrm{m}^{-2}\right)$; III, 中密度 $\left(4-8\right.$ bundle $\left.\cdot \mathrm{m}^{-2}\right) ; \mathrm{IV}$, 小密度 $(<4$ bundle $\cdot \mathrm{m}^{-2}$ )。

Fig. 1 Change of leaf morphological traits and soil physical and chemical properties of Achnatherum splendens on different densities (mean $\pm S E$ ). Different lowercase letters indicate significant differences of different density treatments $(p<0.05)$. I, high density $\left(>12\right.$ bundle $\left.\cdot \mathrm{m}^{-2}\right)$; II, medium density (8-12 bundle $\left.\cdot \mathrm{m}^{-2}\right)$; III, medium density (4-8 bundle $\left.\cdot \mathrm{m}^{-2}\right)$; IV, low density $\left(<4\right.$ bundle $\left.\cdot \mathrm{m}^{-2}\right)$.

$\mathrm{ms} \cdot \mathrm{cm}^{-1}$ 增加到低密度(IV)的 $10.90 \mathrm{~ms} \cdot \mathrm{cm}^{-1}$, 增加了 2.47倍; 土壤含水量呈逐渐减小趋势、从高密度(I) 到低密度(IV)减小了 $71.2 \%$ 。无论是用叶面积, 还是 叶干质量表示叶大小, 不同密度生境叶大小和叶脉 密度均呈“此消彼长”的权衡关系。

\section{3 不同密度条件下湿地芨芨草叶面积、叶干质量} 与叶脉密度的关系

2.3.1 不同密度条件下湿地芨芨草叶面积与叶脉密 度的关系

如图2所示, 芨芨草叶面积与叶脉密度在高密 度(I)和低密度 $(\mathrm{IV})$ 呈极显著负相关关系 $\left(y_{\mathrm{I}}=-2.37 x\right.$ $+1.61, R^{2}=0.88, p<0.01 ; y_{\mathrm{IV}}=-1.92 x+1.47, R^{2}=$
$0.85, p<0.01)$, 在中密度(II、III)呈显著负相关关系 $\left(y_{\mathrm{II}}=-2.25 x+1.47, R^{2}=0.78, p<0.05 ; y_{\mathrm{III}}=-2.17 x+\right.$ $\left.1.54, R^{2}=0.75, p<0.05\right)$; 随着株从密度逐渐降低, 芨芨草叶脉密度和叶面积回归方程的SMA斜率分别 为 $-2.37(95 \%$ 的置信区间 $C I=(-2.55,-2.27)) 、-2.25$ $(95 \%$ 的置信区间 $C I=(-2.36,-2.18)),-2.17$ (95\%的置 信区间 $C I=(-2.27,-1.94))$ 和 $-1.92(95 \%$ 的置信区间 $C I=(-2.19,-1.70))$, 不同密度间二者SMA斜率均与 -1 存在极显著差异 $(p<0.01)$, 且呈逐渐减小趋势。 表明随着株从密度的降低, 叶面积越小, 叶脉密度 越大, 叶面积的生长速率显著小于叶脉密度的增长 速度, 且在不同密度生境呈现不同的权衡关系。 


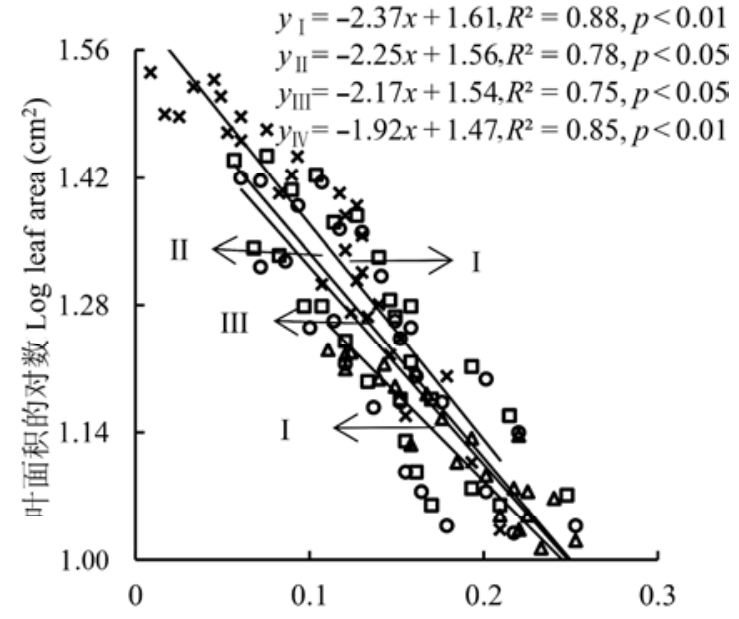

叶脉密度的对数 Log vein density $\left(\mathrm{mm} \cdot \mathrm{mm}^{-2}\right)$

图2 不同密度条件下芨芨草叶面积与叶脉密度的关系。I, 高密度 $\left(>12\right.$ bundle $\left.\cdot \mathrm{m}^{-2}\right)$; II, 中密度(8-12 bundle $\left.\cdot \mathrm{m}^{-2}\right)$; III, 中 密度 $\left(4-8\right.$ bundle $\left.\cdot \mathrm{m}^{-2}\right)$; IV, 低密度 $\left(<4\right.$ bundle $\left.\cdot \mathrm{m}^{-2}\right)$ 。

Fig. 2 Relationship between leaf area and vein density of Achnatherum splendens under different levels of densities. I, high density ( $>12$ bundle $\cdot \mathrm{m}^{-2}$ ); II, medium density (8-12 bundle $\cdot \mathrm{m}^{-2}$ ); III, medium density (4-8 bundle $\left.\cdot \mathrm{m}^{-2}\right)$; IV, low density $\left(<4\right.$ bundle $\left.\cdot \mathrm{m}^{-2}\right)$.

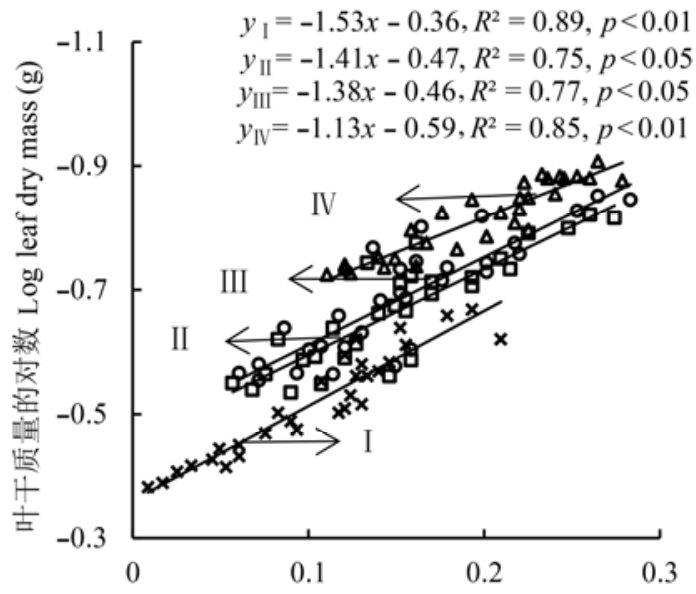

叶脉密度的对数 $\log$ vein density $\left(\mathrm{mm} \cdot \mathrm{mm}^{-2}\right)$

图3 不同密度条件下芨芨草叶干质量与叶脉密度的关系。 $\mathrm{I}$, 高密度 $\left(>12\right.$ bundle $\left.\cdot \mathrm{m}^{-2}\right)$; II, 中密度 $\left(8-12\right.$ bundle $\left.\cdot \mathrm{m}^{-2}\right)$; III, 中密度 $\left(4-8\right.$ bundle $\left.\cdot \mathrm{m}^{-2}\right) ; \mathrm{IV}$, 低密度 $\left(<4\right.$ bundle $\left.\cdot \mathrm{m}^{-2}\right)$ 。

Fig. 3 Relationship between leaf dry mass and vein density of Achnatherum splendens under different levels of densities. I, high density $\left(>12\right.$ bundle $\cdot \mathrm{m}^{-2}$ ); II, medium density (8-12 bundle $\cdot \mathrm{m}^{-2}$ ); III, medium density ( $4-8$ bundle $\cdot \mathrm{m}^{-2}$ ); IV, low density $\left(<4\right.$ bundle $\left.\cdot \mathrm{m}^{-2}\right)$.

\subsection{2不同密度条件下湿地芨芨草叶干质量与叶脉} 密度的关系

如图3所示, 芨菆草叶脉密度与叶干质量在高 密度 $(\mathrm{I})$ 和低密度 (IV) 呈极显著异速负相关 $\left(y_{\mathrm{I}}=\right.$ $-1.53 x-0.36, R^{2}=0.89, p<0.01 ; y_{\mathrm{IV}}=-1.13 x-0.59$,
$\left.R^{2}=0.85, p<0.01\right)$, 在中密度(II、III)呈显著负相关 $\left(y_{\mathrm{II}}=-1.41 x-0.47, R^{2}=0.75, p<0.05 ; y_{\mathrm{III}}=-1.38 x-\right.$ $\left.0.36, R^{2}=0.77, p<0.05\right)$; 随着株从密度的逐渐降 低, 芨芨草叶干质量和叶脉密度回归方程的SMA斜 率分别为 $-1.53(95 \%$ 的置信区间 $C I=(-1.88$, $-1.36)$ )、 -1.41 (95\%的置信区间 $C I=(-1.48,-1.26))$ 、 $-1.38(95 \%$ 的置信区间 $C I=(-1.49,-1.25))$ 和 -1.13 (95\%的置信区间 $C I=(-1.23,-1.01))$, 叶干质量和叶 脉密度在不同密度的 SMA 斜率均与-1存在显著差 异 $(p<0.05)$, 且呈逐渐减小趋势。表明随着密度的 降低, 叶干质量越小、叶脉密度越大, 叶干质量的增 加速率小于叶脉密度的生长速率, 密度生境中资源 的限制加剧了菆芨草叶脉密度和叶干质量的资源权 衡机制。

\section{3 讨论}

植物的生物量分配格局和生理活性对植株密度 的变化具有敏锐的响应性(Cipollini \& Bergelson, 2001; 黎磊和周道玮, 2011), 植物个体往往通过调 整光合构件的生长速率等长期的表型可塑性来提高 植物异质性资源的利用效率(武高林等, 2008)。叶片 是植物碳水耦合权衡的重要器官, 叶大小和叶脉密 度的权衡机制体现了叶性状对不同密度制约、邻体 干扰等过程中的异质性资源的权衡和组合，从而响 应和适应植物的光合蒸腾生理需求, 进而达到生存 和生长之间的经济平衡(Funk \& Vitousek, 2007)。本 研究发现, 高密度(I)和低密度样地(IV)芨芨草的叶 大小与叶脉密度之间存在相反的异速生长关系, 且 均达到极显著性水平 $(p<0.01)$, 中密度样地(II、III) 是芨芨草叶大小逐步减小、叶脉密度逐步增大的过 渡区域, 叶大小和叶脉密度仅呈显著负相关关系 $(p$ $<0.05$ )(图1-3), 与Sack等(2012)对485个物种的叶脉 密度和叶大小关系的研究结果基本一致。不同密度 条件下芨䓅草叶大小和叶脉密度生长关系的差异 性, 既是密度制约对叶片形态和功能的选择塑造, 也是湿地植物根据自身的生存需求, 在碳投入-收 益的权衡下选择的最佳资源配置模式。

植物叶片构型构建与植物生理功能所需的光 照、碳投入和水分运输的投资收益相契合，是植物 提高光合收益的一个重要的生活史策略(Westoby et al., 2002; 孙素静等, 2015)。种群密度的制约性调节 是决定植物生物量分配格局的关键因素(黎磊和周 
道玮, 2011), 能够促使植物权衡有限光合产物在叶 肉、叶脉等组织间的合理分配(Uhl \& Mosbrugger, 1999), 以期实现光合碳获取最大化的目标。研究区 属于河西走廊温带干旱区的天然湿地生态系统, 土 壤水和地下水是芨芨草可能的主要利用水源(吴华 武等, 2015)。处于湿地群落高密度(I)样地的芨芨草 种群占有的地上环境空间相对窄, 为了保证有限空 间下光拦截能力和光合碳获取能力最大化, 芨芨草 以牺牲叶脉密度的资源分配为代价, 选择了增大叶 面积和叶干质量的权衡策略和叶片构型构建机制 (图1-3)。主要原因有: (1)来自邻株植物的遮阴改变 了植物所处光环境的红外/远红外光比率 $(R / F R)$, 限 制了可获得性PAR (表1), 芨芨草做出增加株高、减 少分枝数的遮阴躲避反应(表1), 将更多的生物量投 资于单位分枝上的叶片, 形成最大的叶面积和叶肉 组织密度, 从而减弱了相邻植株的光抑制作用, 保 证了最大的光合作用面积和同化速率, 能够将更多 的光能转化为用于自身生长的化学能(黎磊和周道 玮, 2011); (2)较高的株从密度避免了土壤水分的潜 在蒸散(吴华武等, 2015), 土壤含水量较高且盐分胁 迫弱(图1), 䓅芨草进行光合作用的水分输送阻力较 小, 较小的叶脉密度也能满足植株较低的光合和蒸 腾速率的水分需求(表1), 将更多的生物量用于光转 换和同化结构的构建能够极大地缓冲资源有限性约 束; (3)该生境芨芨草比叶面积最大(图1), 说明低光 胁迫下菆芨草种群为了捕捉更多的光能会倾向用有 限的营养物质创造出更大的受光面积以利吸收更多 光能, 最大限度地获取光照和控制水分散失之间的 平衡(Wright et al., 2004)。因此, 高密度(I)条件下夰 菆草叶脉密度和叶大小形成了极显著负相关关系 $(p$ $<0.01)$ 。与West等(1997)提出的“自然选择的压力必 然使其向最优化的方向进化, 使得与外界环境的交 换面积和新陈代谢效率最大化, 并使营养物质运输 距离和时间最小化”一致。

最优化分配理论预测, 在无邻体植物竞争时, 植物应该增大获取限制资源的器官分配, 而减少获 取非限制资源的器官分配(Weiner, 2004), 即在特定 的环境下, 植物为了满足生存需求会形成不同的形 态结构和特定的水分供应策略, 使植物更好地调整 和适应异质性资源(龚容和高琼, 2015)。湿地芨芨草 种群处于较低密度(IV)样地时, 生存空间扩大、光合 有效辐射最大(表1)、伴随着较高的光合速率和蒸腾
速率(表1), 为了进一步拓展生存生态位, 芨芨草优 先倾向于增加分枝数(表1), 从而选择了减少单位分 枝上叶面积和叶干质量的资源投资, 将更多的光合 产物用于增加叶脉密度以保证高效率的水分输导系 统的生存模式(图1-3)。主要原因有: (1)夰芨草为了 避免较强的蒸腾作用引起自身失水过多而大量减小 叶面积(图1), 这不仅达到了小叶易进行热量和物质 交换的目的, 而且减小了蒸腾作用面积, 有利于保 持生命活动的水分平衡(党晶晶等, 2014), 形成了最 小的叶面积和叶干质量(图1); (2)该生境中土壤含水 量最低、土壤电导率大(图1), 盐分胁迫造成的“生理 干旱”现象阻碍了植物的吸水作用(周洪华和李卫 红，2015), 芨芨草将更多的叶生物量投资于机械支 持与微管结构, 使得该生境的芨芨草叶脉密度较高 密度(I)增加了 $13.33 \%$ (图1), 为水分转移至叶肉提 供更大的接触面积和便捷的路径, 因此增加叶片中 水分运输的量与速率也许是在该生境维持生存繁衍 的关键性策略(Nardini et al., 2010); (3)该生境芨芨 草比叶面积较高密度(I)减小了 $12.5 \%$ (图1), 即当土 壤含水量不足、叶片蒸腾速率较大时, 芨芨草选择 较小的叶面积有利于植物获取异质性资源、提高适 应贫㾑环境的能力和强光下的自我保护构造 (Wright et al., 2004), 这是湿地芨芨草对环境胁迫所 产生的一种安全性经济权衡策略。因此, 低密度(IV) 样地芨芨草的叶脉密度和叶大小呈极显著的负相关 关系 $(p<0.01)$, 这与Sack等(2012)关于 “小叶中高的 叶脉密度提供冗余的“高速公路”来运输水分, 有利 于提高植物的耐旱能力, 增强叶片抵御虫食干扰能 力”的研究结果一致。

植物个体间的相互作用实际上是可获得性资源 的有限性导致的构件水平的竞争(黎磊和周道玮, 2011), 在不同密度的资源竞争强度下, 植物的表型 可以通过改变不同器官间生物量分配的可塑性反应 进行调整(Cheplick, 2006)。芨芨草种群处于中密度 (II、III)样地时, 地表土壤理化过程和强度均发生了 变化(图1), 种内对空间和光的竞争有所减弱, 株高 居中, 芨芨草分枝数、净光合和蒸腾速率最大 (表1), 为了增强资源竞争能力和生境适合度, 芨芨草选择 了折中光合面积和同化结构的稳步投资, 兼顾了植 株的光截取和水分输送等功能需求, 选择了叶大小 和叶脉密度均衡生长模式, 可以达到更大限度占有 地上部分资源和拓展空间的目的, 同时也能减少植 
株内部的资源竞争, 这对于种群的稳定与扩张都是 有利的。因此, 生长在中密度(II、III)样地的芨芨草 叶大小和叶脉密度的相关性仅达到显著水平 $(p<$ 0.05 , 图2, 图3), 是叶大小由大到小、叶脉密度由小 到大转变的区域, 这是由于光合产物在不同构件或 功能间权衡分配的结果。

植物叶大小和叶脉密度关系不仅是植物个体生理 过程的表现, 也体现了植物叶形态的微生境依赖规律 及表型可塑性。湿地群落中高密度(I)样地的芨芨草种 群为了适应强烈的光竞争, 权衡叶片组织和水分运 输系统的碳投入, 选择减少叶脉密度而增加叶大小 资源投资比例的异速生长格局, 以保证稳定的光合 收益; 低密度(IV)样地的芨芨草趋向于以牺牲叶大 小为代价构建较大的叶脉密度, 保证畅通高效的水 分运输路径应对水分胁迫和增加自身的竞争力; 不 同种群密度所选择的生存模式均体现了重要的碳水投资器官如何有效调节资源分配, 即水分运输能 力和光合能力的匹配问题。这种随着环境变化的感应 机制和“趋利避害”的表型可塑性是湿地植物与环境长 期相互协同进化与适应的结果, 可以提高密度制约下 植物的生存、生长和繁殖适合度, 也是顺利完成生活 史周期的最佳生理和功能状态。本文仅从密度角度 分析了湿地生态系统中植物叶大小-叶脉密度的变 化特征, 它们的关系可能还受到地下生物量、光合 色素含量和物种遗传特性等其他因子的影响, 还需 要进一步的实验验证。

\section{基金项目 国家自然科学基金(41461013 和 91125014)。}

\section{参考文献}

Berlyn GP, Miksche JP (1976). Botanical Microtechnique and Cytochemistry. Iowa State University Press, Ames, USA.

Brodribb TJ, Feild TS, Sack L (2010). Viewing leaf structure and evolution from a hydraulic perspective. Functional Plant Biology, 37, 488-498.

Brodribb TJ, Jordan GJ (2011). Water supply and demand remain balanced during leaf acclimation of Nothofagus cunninghamii trees. New Phytologist, 192, 437-448.

Castro-Díez P, Puyravaud JP, Cornelissen JHC (2000). Leaf structure and anatomy as related to leaf mass per area variation in seedlings of a wide range of woody plant species and types. Oecologia, 124, 476-486.

Cheplick GP (2006). A modular approach to biomass allocation in an invasive annual (Microstegium vimineum; Poaceae). American Journal of Botany, 93, 539-545.
Cipollini DF, Bergelson J (2001). Plant density and nutrient availability constrain constitutive and wound-induced expression of trypsin inhibitors in Brassica napus. Journal of Chemical Ecology, 27, 593-610.

Dang JJ, Zhao CZ, Li Y, Hou ZJ, Dong XG (2014). Variations with slope in stem and leaf traits of Melica przewalskyi in alpine grassland. Chinese Journal of Plant Ecology, 38, 1307-1314. (in English with Chinese abstract) [党晶晶, 赵成章, 李钰, 侯兆疆, 董小刚 (2014). 高寒草地甘肃 臭草茎-叶性状的坡度差异性. 植物生态学报, 38, 1307-1314]

Falster DS, Warton DI, Wright IJ (2012). User's Guide to SMATR: Standardised Major Axis Tests \& Routines Version 2.0. http://www.bio.mq.edu.au/ecology/SMATR/. Cited: 2014-03-11.

Funk JL, Vitousek PM (2007). Resource-use efficiency and plant invasion in low-resource systems. Nature, 446, 1079-1081.

Gong R, Gao Q (2015). Research progress in the effects of leaf hydraulic characteristics on plant physiological functions. Chinese Journal of Plant Ecology, 39, 300-308. (in English with Chinese abstract) [龚容, 高琼 (2015). 叶片结 构的水力学特性对植物生理功能影响的研究进展. 植 物生态学报, 39, 300-308.]

Harvey PH, Pagel MD (1991). The Comparative Method in Evolutionary Biology. Oxford University Press, Oxford, UK.

Japhet W, Zhou DW, Zhang HX, Zhang HX, Yu T (2009). Evidence of phenotypic plasticity in the response of Fagopyrum esculentum to population density and sowing date. Journal of Plant Biology, 52, 303-311.

Jin Y, Wang CK (2015). Trade-offs between plant leaf hydraulic and economic traits. Chinese Journal of Plant Ecology, 39, 1021-1032. (in Chinese with English abstract) [金鹰, 王传宽 (2015). 植物叶片水力与经济性状权衡关系的 研究进展. 植物生态学报, 39, 1021-1032.]

Li L, Zeng H, Guo DL (2013). Leaf venation functional traits and their ecological significance. Chinese Journal of Plant Ecology, 37, 691-698. (in English with Chinese abstract) [李乐, 曾辉, 郭大立 (2013). 叶脉网络功能性状及其 生态学意义. 植物生态学报, 37, 691-698.]

Li L, Zhou DW (2011). Density-dependent regulation of aboveand below-ground modules in Allium cepa var. proliferum populations. Chinese Journal of Plant Ecology, 35, 284-293. (in English with Chinese abstract) [黎磊, 周道 玮 (2011). 红葱种群地上和地下构件的密度制约调节. 植物生态学报, 35, 284-293.]

Liu MH, Xin ZM, Xu J, Sun F, Dou LJ, Li YH (2013). Influence of leaf size of plant on leaf transpiration and temperature in arid regions. Chinese Journal of Plant Ecology, 37, 436-442. (in English with Chinese abstract) [刘明虎, 
辛智鸣, 徐军, 孙非, 窦立军, 李永华 (2013). 干旱区 植物叶片大小对叶表面蒸腾及叶温的影响. 植物生态 学报, 37, 436-442.]

Mcdonald PG, Fonseca CR, Overton JM, Westoby M (2003). Leaf-size divergence along rainfall and soil-nutrient gradients: Is the method of size reduction common among clades? Functional Ecology, 17, 50-57.

Nardini A, Raimondo F, Lo Gullo MA (2010). Leafminers help us understand leaf hydraulic design. Plant, Cell \& Environment, 33, 1091-1100.

Niklas KJ, Cobb ED, Niinemets Ü, Reich PB, Sellin A, Shipley B, Wright IJ (2007). "Diminishing returns” in the scaling of functional leaf traits across and within species groups. Proceedings of the National Academy of Sciences of the United States of America, 104, 8891-8896.

Pitman EJG (1939). A note on normal correlation. Biometrika, 31, 9-12.

Reich PB, Cornelissen H (2014). The world-wide 'fast-slow' plant economics spectrum: A traits manifesto. Journal of Ecology, 102, 275-301.

Ren QJ, Li HL, Bu HY (2015). Comparison of physiological and leaf morphological traits for photosynthesis of the 51 plant species in the Maqu alpine swamp meadow. Chinese Journal of Plant Ecology, 39, 593-603. [任青吉, 李宏林, 卜海燕 (2015). 玛曲高寒沼泽化草甸51种植物光合生 理和叶片形态特征的比较. 植物生态学报, 39, 593-603.]

Sack L, Frole K (2006). Leaf structural diversity is related to hydraulic capacity in tropical rain forest trees. Ecology, 87, 483-491.

Sack L, Scoffoni C (2013). Leaf venation: Structure, function, development, evolution, ecology and applications in the past, present and future. New Phytologist, 198, 983-1000.

Sack L, Scoffoni C, McKown AD (2012). Developmentally based scaling of leaf venation architecture explains global ecological patterns. Nature Communications, 3, 837.

Shi YC, Zhao CZ, Song QH, Du J, Chen NJ, Wang JW (2015). Slope-related variations in twig and leaf traits of Robinia pseudoacacia in the northern mountains of Lanzhou. Chinese Journal of Plant Ecology, 39, 362-370. (in English with Chinese abstract) [史元春, 赵成章, 宋清华, 杜晶, 陈静, 王继伟 (2015). 兰州北山刺槐枝叶性状的坡向差 异性. 植物生态学报, 39, 362-370.]

Silvertown J, Charlesworth D (2001). Introduction to Plant Population Biology. Blackwell, London.

Sun SJ, Li FL, Bao WK (2015). Advances on construction of leaf venation system and its significance of phylogeny. Journal of Tropical and Subtropical Botany, 23, 353-360. (in English with Chinese abstract) [孙素静, 李芳兰, 包维 楷 (2015). 叶脉网络系统的构建和系统学意义研究进 展. 热带亚热带植物学报, 23, 353-360.]
Uhl D, Mosbrugger V (1999). Leaf venation density as a climate and environmental proxy: A critical review and new data. Palaeoecology, 149, 15-26.

Wang CS, Wang SP (2015). A review of research on responses of leaf traits to climate change. Chinese Journal of Plant Ecology, 39, 206-216. (in Chinese with English abstract) [王常顺, 汪诗平 (2015). 植物叶片性状对气候变化的 响应研究进展. 植物生态学报, 39, 206-216.]

Wang J, Cheng JM, Wan HE, Fang F (2004). Study on soil moisture characteristics and water use efficiency of Achnatherum splendens grassland in Loess Plateau. Arid Meteorology, 22, 51-55.

Warton DI, Weber NC (2002). Common slope tests for bivariate errors-in-variables models. Biometrical Journal, 44, 161-174.

Warton DI, Wright IJ, Falster DS, Westoby M (2006). Bivariate line-fitting methods for allometry. Biological Reviews, 81, 259-291.

Weiner J (2004). Allocation, plasticity and allometry in plants. Perspectives in Plant Ecology, Evolution and Systematics, 6, 207-215.

West GB, Brown JH, Enquist BJ (1997). A general model for the origin of allometric scaling laws in biology. Science, 276, 122-126.

Westoby M, Falster DS, Moles AT (2002). Plant ecological strategies: Some leading dimensions of variation between species. Annual Review of Ecology Systematics, 33, 125-159.

Wright IJ, Reich PB, Westoby M, Ackerly DD, Baruch Z, Bongers F, Cavender-Bares J, Chapin T, Cornelissen JH, Diemer M, Flexas J, Garnier E, Groom PK, Gulias J, Hikosaka K, Lamont BB, Lee T, Lee W, Lusk C, Midgley JJ, Navas ML, Niinemets U, Oleksyn J, Osada N, Poorter H, Poot P, Prior L, Pyankov VI, Roumet C, Thomas SC, Tjoelker MG, Veneklaas EJ, Villar R (2004). The worldwide leaf economics spectrum. Nature, 428, 821-827.

Wu GL, Chen M, Du GZ (2008). Effects of nutrient and lighton seedlings morphological plasticity of four Saussurea species with different ecological breadth. Chinese Journal of Applied Ecology, 19, 1708-1713. (in Chinese with English abstract) [武高林, 陈敏, 杜国祯 (2008). 营养 和光照对不同生态幅风毛菊属植物幼苗形态可塑性的 影响. 应用生态学报, 19, 1708-1713.]

Wu HW, Li XY, Jing ZY, Li J, Zheng XR, Zhao DZ (2015). Variations in water use for Achnatherum splendens in Lake Qinghai watershed based on $\delta \mathrm{D}$ and $\delta^{18} \mathrm{O}$. Acta Ecologica Sinica, 35, 8174-8183. (in English with Chinese abstract) [吴华武, 李小雁, 蒋志云, 李静, 郑肖 然, 赵殿智 (2015). 基于 $\delta \mathrm{D} 、 \delta^{18} \mathrm{O}$ 的青海湖流域芨苃草 水分利用来源变化研究. 生态学报, 35, 8174-8183.]

Wu ZS, Guo BZ (1989). Flora Repubulicae Popularis Sinicae. (Tomus 9). Science Press, Beijing. 1087. (in Chinese) [吴

www.plant-ecology.com 
征镒, 郭本兆 (1989). 中国植物志(第九卷). 科学出版 社, 北京. 1087.]

Yang DM, Zhang JJ, Zhou D, Qian MJ, Zheng Y, Jin LM (2012). Leaf and twig functional traits of woody plants and their relationships with environmental change. Chinese Journal of Ecology, 31, 702-713. (in English with Chinese abstract) [杨冬梅, 章佳佳, 周丹, 钱敏杰, 郑瑶, 金灵 妙 (2012). 木本植物茎叶功能性状及其关系随环境变 化的研究进展. 生态学杂志, 31, 702-713.]

Zhan YF, Ma L, Li XY, Teng YF, Lu YF, Tian XP (2012). Niches of dominant populations of wetland plants in Zhangye section of Heihe River. Journal of Northeast Forestry University, 40(10), 61-66. (in English with Chinese abstract) [占玉芳, 马力, 李小燕, 滕玉风, 鲁延 芳, 田晓萍 (2012). 黑河流域(张掖段)湿地植物群落优 势种群生态位. 东北林业大学学报, 40(10), 61-66.]

Zhang MJ, Liu MS, Xu C, Chi T, Hong C (2012). Spatial pattern responses of Achnatherum splendens to environmental stress in different density levels. Acta Ecologica Sinica, 32, 595-604. (in English with Chinese abstract) [张明娟, 刘茂松, 徐驰, 池婷, 洪超 (2012). 不同密度条件下芨夰草空间格局对环境胁迫的响应. 生态学报, 32, 595-604.]

Zhang SB, Guan ZJ, Sun M, Zhang JJ, Cao KF, Hu H (2012).
Evolutionary association of stomatal traits with leaf veindensity in Paphiopedilum, Orchidaceae. PLOS ONE, 7, e40080. doi: 10.1371/journal.pone.0040080.

Zhang YF, Wang W, Liang CZ, Wang LX, Pei H, Wang CY, Wang WF (2012). Suitable habitat for the Achnatherum splendens community in typical steppe region of Inner Mongolia. Acta Ecologica Sinica, 32, 1193-1201. (in English with Chinese abstract) [张翼飞, 王炜, 梁存柱, 王立新, 裴浩, 王成燕, 王伟峰 (2012). 内蒙古典型草 原区芨䓅草群落适生生境. 生态学报, 32, 1193-1201.]

Zhang YQ, Liang CZ, Wang W, Wang LX, Peng JT, Yan JC, Jia JC (2010). Soil salinity and Achnatherum splendens distribution. Chinese Journal of Ecology, 29, 2438-2443. (in English with Chinese abstract) [张雅琼, 梁存柱, 王 炜, 王立新, 彭江涛, 间建成, 贾成朕 (2010). 芨芨草 群落土壤盐分特征. 生态学杂志, 29, 2438-2443.]

Zhou HH, Li WH (2105). Responses and adaptation of xylem hydraulic conductivity to salt stress in Populus euphratica. Chinese Journal of Plant Ecology, 39, 81-91. (in English with Chinese abstract) [周洪华, 李卫红 (2015). 胡杨木 质部水分传导对盐胁迫的响应与适应. 植物生态学报, 39, 81-91.]

责任编委: 常 杰 责任编辑: 李 敏

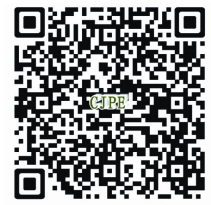

植物生态学报官网

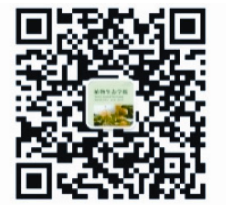

微信订阅号

期刊及学科

相关信息发布

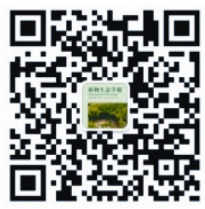

微信服务号

稿件状态查询

全文检索汶览 